\title{
Neutrophil extracellular traps in
}

\section{children with meningococcal sepsis}

Tamara Hoppenbrouwers, MSc*1,2 ${ }^{*}$ Navin P. Boeddha, MD*3,4, Ebru Ekinci ${ }^{4}$, Marieke Emonts, $\mathrm{PhD}^{5,6}$ ,Jan A. Hazelzet, Prof ${ }^{7}$, Gertjan J. Driessen, $\mathrm{PhD}^{* * 4,8}$,Moniek P. de Maat, PhD**2

Running title: NETs in meningococcal sepsis

1) Department of Plastic and Reconstructive Surgery, Erasmus University Medical Center, Rotterdam The Netherlands

2) Department of Hematology, Erasmus University Medical Center, Rotterdam, The Netherlands

3) Intensive Care and Department of Pediatric Surgery, Sophia Children's Hospital, Erasmus University Medical Center, Rotterdam, The Netherlands

4) Department of Pediatrics, Division of Pediatric Infectious Diseases \& Immunology, Sophia Children's Hospital, Erasmus University Medical Center, Rotterdam, The Netherlands

5) Paediatric Infectious Diseases and Immunology Department, Great North Children's Hospital, Newcastle upon Tyne Hospitals NHS Foundation Trust, Newcastle upon Tyne, United Kingdom 6) Institute of Cellular Medicine, Newcastle University, Newcastle upon Tyne, United Kingdom

7) Department of Public Health, Erasmus University Medical Center, Rotterdam, The Netherlands 8) Department of Paediatrics, Juliana Children's Hospital/Haga Teaching Hospital, The Hague, The Netherlands

\footnotetext{
* These authors have contributed equally to the work presented in this manuscript.

** These authors have contributed equally to the work presented in this manuscript
} 


\section{Corresponding author:}

Dr. M.P.M. de Maat

Dept Hematology, Nb845a

Erasmus University Medical Center

P.O. Box 2040

3000 CA Rotterdam

The Netherlands

Tel. +31 107033442

Email: $\underline{\text { m.demaat@erasmusmc.nl }}$

\section{Conflict of Interest:}

The authors declare no conflict of interest.

\section{Financial support:}

Research leading to these results has received funding from the European Union's seventh Framework program under EC-GA no. 279185 (EUCLIDS). Also, research has been supported, in part, by an unrestricted grant from Baxter Bioscience, Vienna, Austria, in 2000. These funders had no role in study design, data collection and analysis, decision to publish, or preparation of the manuscript. 


\begin{abstract}
Objective

Children with meningococcal sepsis are highly at risk for fulminant disease, multi-organ failure and death. Recently, neutrophil extracellular traps (NETs) levels have been indicated as a marker for severity in different kinds of sepsis. Our aim was to study the role of NETosis in meninogococcal sepsis in children.
\end{abstract}

Design and setting

We measured MPO-DNA, a marker for NETs, in serum of meningococcal sepsis patients upon admission to PICU, at 24 hours, and at 1 month and studied the association with clinical outcome. Subsequently, we tested whether N. meningitidis, isolated from children with meningococcal sepsis, were able to induce NETosis, using confocal microscopy live imaging.

\title{
Patients
}

We included exclusively children with meningococcal sepsis.

Interventions

From each patient, serum was collected for analysis.

\section{Measurements}

We used ELISAs to measure MPO-DNA in patients serum. We also included inflammatory markers that were previously measured in this group.

Main results

MPO-DNA levels at admission ( $n=35$, median $0.21 \mathrm{AU} / \mathrm{mL}$, IQR $0.12-0.27)$ and at 24 hours $(n=39$, 
median $0.14 \mathrm{U} / \mathrm{mL}$, IQR 0.09-0.25) were significantly higher than the MPO-DNA levels after 1 month (controls, $\mathrm{n}=36$, median $0.07 \mathrm{AU} / \mathrm{mL}, \mathrm{QQR} 0.05-0.09, \mathrm{p}<0.001$ ). We did not observe a correlation between MPO-DNA levels and mortality, cell-free DNA or other inflammatory markers. In addition, $N$. meningitidis are fast and strong inducers of NETosis.

\section{Conclusions}

Children admitted to PICU for meningococcal sepsis have higher NETs levels at admission and after 24 hours than controls. NETs levels were not associated with outcome, cell-free DNA or other inflammatory markers. These NETs may be induced by N. meningitidis, since these are strong NETosis inducers.

\section{Keywords:}

NETs; Meningococcal infections; N. meningitidis; Critical care; Pediatrics; Mortality 


\section{Introduction}

Meningococcal sepsis is notorious for its rapid progression to fulminant disease, multi-organ failure and death ${ }^{1,2}$. Complex interplays between host, pathogen, and environmental factors, including immune evasion mechanisms, determine the severity of Neisseria meningitidis infections, ranging from harmless colonization to lethal disease ${ }^{3-5}$.

A recently identified mechanism that may play a role in the pathology of meningococcal sepsis is NETosis. The release of neutrophil extracellular traps (NETs) is an important part of innate immune defense ${ }^{6,7}$. NETs are an extracellular DNA matrix, containing also granule proteins and histones, released by neutrophils to degrade virulence factors and to kill bacteria ${ }^{8}$. NETs are primarily considered as a protective mechanism against a broad range of microorganisms, including gramnegative and gram-positive bacteria, because they prevent bacteria from spreading and contain toxic histones ${ }^{8,9}$. Several inducers of NETosis are known, including bacterial species such as Staphylococcus aureus ${ }^{10}$. Multiple studies describe a negative influence on outcome in mice and humans ${ }^{11,12}$, since the tissue damage caused by NETs may contribute to disease severity ${ }^{13,14}$. Also cell free DNA ( $c f D N A$ ), the backbone of a NET, has been described to contribute to disease via the formation of microthrombi and to inhibit fibrinolysis ${ }^{15}$.

Studies on the relationship between NETs and Neisseria meningitidis in sepsis are lacking. Our objective was to study the role of NETs in children with meningococcal sepsis. We measured levels of NETs, cell-free DNA and other inflammatory markers in serum from children with meningococcal sepsis and studied the association with severity of disease. We also investigated whether $N$. meningitidis isolates from patients are able to induce NETosis. 


\section{Material and methods}

\section{Patients and samples}

From 1988 to 2005 , children with meningococcal sepsis presenting to the pediatric intensive care unit (PICU) of Erasmus MC-Sophia Children's Hospital (Rotterdam, The Netherlands) were prospectively enrolled in meningococcal studies ${ }^{16-19}$. These studies were conducted in accordance with the Declaration of Helsinki and Good Clinical Practice guidelines. All individual meningococcal studies as well as the current laboratory study (MEC-2015-497) were approved by the ethical committee of Erasmus MC, and written informed consent was obtained from parents or legal guardians. All patients fulfilled internationally agreed criteria for sepsis ${ }^{20}$. Blood samples were taken on admission to PICU, at 24 hours after PICU admission, and at 1 month after PICU admission. Since all children were healthy at 1 month, the 1 month convalescence samples were used as controls. Samples were processed on ice and serum was stored at $-80^{\circ} \mathrm{C}$ until analysis. This is a retrospective laboratory study in remaining samples of prospectively collected samples.

\section{Clinical data collection}

Clinical data were collected prospectively. Disease severity was indicated by Pediatric Risk of Mortality (PRISM) ${ }^{21}$, predicted death based on the Rotterdam score ${ }^{17}$, disseminated intravascular coagulation (DIC) score ${ }^{22}$, and the base excess and platelet count at presentation (BEP) score ${ }^{23}$. Patients were classified as death if death occurred during PICU-stay. PICU-free days in patients who died were considered zero.

\section{NETosis measurements}

Our MPO-DNA ELISA assay was performed as reported earlier ${ }^{24}$. For detection of NETs in serum, we adjusted the commercial human ELISA kit that measures cell death (Cell death detection ELISA ${ }^{\text {PLUS }}$, 
Cat. No 11920685001, Roche Diagnostics Nederland B.V., Almere, the Netherlands) ${ }^{25}$. Briefly, ELISA plates were coated with a mouse-anti-human myeloperoxidase (MPO) monoclonal antibody (AbD Serotec, Oxford, UK), a NETs marker, overnight at $4{ }^{\circ} \mathrm{C}$. The plates were then washed with Phosphate Buffered Saline (PBS) containing 0,05\% v/v Tween ${ }^{\circledR}-20$ and incubated with blocking solution (1\% BSA/PBS) overnight at $4{ }^{\circ} \mathrm{C}$. Next, samples were added to the plate and incubated with MPO-DNA immunoreagens for 2 hours at 300 rpm at RT, washed and incubated with 2,2'-azino-bis(3ethylbenzothiazoline-6-sulphonic acid) (ABTS) reagens for $30 \mathrm{~min}$ at $250 \mathrm{rpm}$ at RT. The reaction was stopped with stop solution from the kit and plates were measured using a Biotek reader (FLX 800, Austria) at $405 \mathrm{~nm}$ with a $490 \mathrm{~nm}$ reference filter. The NETs reference curve was created from neutrophils that were transferred to FBS serum and stimulated with phorbol-myristate-acetate (PMA) for 4 hours. Samples were serially diluted and stored at $-80^{\circ} \mathrm{C}$. Values are expressed as arbitrary units $(\mathrm{AU} / \mathrm{mL})$.

Inflammatory markers CRP, fibrinogen, soluble TNFr, IL-1B, IL-6, and IL-8 and cell-free DNA (nucleosomes) were measured in previous studies from our research group ${ }^{16-19,26}$.

\section{Neutrophil isolation}

Neutrophils were isolated as previously described ${ }^{10}$. Briefly, red blood cells and granulocytes were isolated from blood from adult healthy donors, as approved by the Medical Ethics Committee of the Erasmus MC, using Lymphoprep ${ }^{\mathrm{TM}}$ (Stem cell Technologies, Grenoble, France). Granulocytes were further purified by lysing the erythrocytes with erythrolysis buffer $\left(3.1 \mathrm{M} \mathrm{NH} \mathrm{Nl}_{4}, 0.2 \mathrm{M} \mathrm{KHCO}, 0.02 \mathrm{M}\right.$ EDTA, pH 7.4) and washing the neutrophil rich pellet twice with HEPES $(0.115 \mathrm{M} \mathrm{NaCl}, 0.012 \mathrm{mM}$ $\mathrm{CaCl}_{2}, 1.51 \mathrm{mM} \mathrm{MgCl}$, $4 \mathrm{mM} \mathrm{KCl}, 0.01 \mathrm{M}$ HEPES, pH 7.4) buffer. 
In vitro NETosis

For experiments with meningococcal bacteria, neutrophils were transferred to Dulbecco's Modified Eagle Medium (DMEM) culture medium without any additions (Biowhittaker, Lonza, Walkersville, USA) in a confocal ring. Propidium lodide (PI, 1:400, Sigma Aldrich, Zwijndrecht, The Netherlands) was added to visualize extracellular DNA during live imaging.

To visualize extracellular DNA (NETs), neutrophils were stained for DNA with Hoechst 34580 (1: 10000, Life Technologies, Landsmeer, The Netherlands) and PI (1:400, Sigma Aldrich, Zwijndrecht, The Netherlands) prior to contact with $N$. meningitidis as described previously by our group ${ }^{27}$. Clinical isolates of $N$. meningitidis bacteria were cultured as described previously ${ }^{28}$. After 4 hours of culturing, $100 \mu \mathrm{l} 1 \times 10^{8} / \mathrm{ml}$ bacteria were added to $500 \mu \mathrm{l} 2 \times 10^{6} / \mathrm{ml}$ neutrophils. NETs were visualized using confocal microscopy (Leica SP5 AOBS). Excitation of Hoechst with a 405 laser and a BP 420-500 emission filer, and excitation of PI with a 561141 and BP 580-620 emission filter. Elongated PI positive structures larger than $10 \mu \mathrm{m}$ (the average diameter of a neutrophil) were defined as NETs. Round PI positive structures equal or smaller than $10 \mu \mathrm{m}$ were defined as necrotic neutrophils.

\section{Statistical analysis}

Patient characteristics and MPO levels are presented as numbers and proportions (means and percentages) or medians and interquartile ranges. To compare MPO-DNA levels between the three time points, we used the Kruskal-Wallis test, including the post-hoc Mann-Whitney rank-sum test. To compare MPO-DNA level between survivors and non-survivors, we used the Mann-Whitney ranksum test. Correlations were assessed using Spearman's rank correlation. Linear regression analyses were performed on logarithmically transformed data. Graphs were created with GraphPad Prism 7.00. All statistical analyses were performed using Statistical Package for the Social Sciences (SPSS, IBM, version 21). Two-sided $p$-values $<0.05$ were considered to indicate statistical significance. 


\section{Results}

\section{NETs levels in meningococcal sepsis}

In this study we included 60 children with meningococcal sepsis ( $58 \%$ male, median age 2 years and 10 months [IQR 21 months-9 years]), of whom 35, 39, and 36 serum samples were available originating from admission to PICU, after 24 hours, and at 1 month, respectively. Patient characteristics are presented in Table 1.

MPO-DNA levels at admission ( $n=35$, median $0.21 \mathrm{AU} / \mathrm{mL}$, IQR $0.12-0.27$ ) and at 24 hours ( $n=39$, median $0.14 \mathrm{AU} / \mathrm{mL}$, IQR 0.09-0.25) were significantly higher than the convalescent MPO-DNA levels in the control group of survivors after 1 month ( $n=36$, median $0.07 \mathrm{AU} / \mathrm{mL}$, IQR $0.05-0.09, p<0.001$ ) (Figure 2A). The MPO-DNA level in children who eventually died did not differ significantly from survivors at admission to PICU (non-survivors: $n=11$, median $0.23 \mathrm{AU} / \mathrm{mL}$, IQR $0.12-0.47$; survivors: $\mathrm{n}=23$, median $0.16 \mathrm{AU} / \mathrm{mL}$, IQR 0.12-0.25, $\mathrm{p}=0.14$ ) and after 24 hours (non-survivors: $\mathrm{n}=3$, median $0.14 \mathrm{AU} / \mathrm{mL}$; survivors: $\mathrm{n}=34$, median $0.15 \mathrm{AU} / \mathrm{mL}$, IQR 0.09-0.26, $\mathrm{p}=0.48$ ) (Figure 2B).

\section{NETs and inflammatory markers in sepsis}

Since NETs can be induced by inflammatory markers, and NETs in turn can lead to an increased inflammatory response, we explored the correlations between inflammatory markers (neutrophil count, and serum C-reactive protein (CRP), fibrinogen, soluble Tumor Necrosis Factor (TNFr), Interleukine (IL)-1B, IL-6 and IL-8 levels) and MPO-DNA levels in our samples. We did not observe any significant correlation of NETs levels with any of these markers (Table 2).

\section{NETs and cell-free DNA}

Because nucleosome levels in serum reflect cell-free DNA, we tested how serum levels of nucleosomes were correlated to serum levels of NETs. We did not find a significant correlation 
between nucleosomes and MPO-DNA at any of the time points $(t=0: r=0.162, p=0.54 ; t=24: r=0.044$, $p=0.86 ; t=1 m: r=0.025, p=0.93)$.

\section{In vitro formation of NETs in the presence of Neisseria meningitidis}

In our pilot experiment, we saw that clinical isolates from N. meningitidis induce NETs in vitro in adult neutrophils. NETs were visible after 15 minutes of incubation of neutrophils with the bacteria (Fig. 1). In the control experiment with unstimulated neutrophils without bacteria, no NETs were seen.

Since neutrophils started dying approximately 30-40 minutes after contact with $N$. meningitidis, we only observed NETs within this time frame. 


\section{Discussion}

This is the first study to describe NETs in children with meningococcal sepsis, describing both in vitro and in vivo data. The main findings of our study arethat NETs levels in these children are higher at admission to PICU and after 24 hours compared to levels after one month. We also found that NETs levels were not associated with clinical outcome in our cohort. Lastly, in a pilot experiment we found that $N$. meningitidis, isolated from children with meningococcal sepsis, are strong NETs inducers in adult neutrophils.

In our study the NETs levels, measured by MPO-DNA levels, in children with meningococcal sepsis are higher in the acute phase of disease, i.e. at admission to PICU and at 24 hours after admission, compared to 1 month. Similar results were previously observed in adult studies, reporting increased NETs levels during the acute phase of sepsis ${ }^{11,13,29}$.

Multiple animal and patient studies associated NETs levels with sepsis severity and outcome ${ }^{13,14}$. This effect of NETs in sepsis likely results from the interplay between NETs, platelets and thrombin, which activates coagulation and inhibits fibrinolysis, leading to more severe DIC ${ }^{13,14,30}$. In our study, we did not find an association between NETs, severity parameters, and outcome. Our study group was relatively small, and may have been underpowered to detect these effect. Alternatively, the positive and negative effects of NETosis, respectively containing meningococcal infection and inducing tissue damage and deregulate coagulation might be balanced in meningococcal sepsis in children.

Previously, Zeerleder et al (2013) ${ }^{31}$ measured nucleosomes in this cohort and reported that nucleosomes are correlated with several factors, such as organ dysfunction, several cytokines and patient outcome. In some studies, nucleosomes are used as marker for NETosis ${ }^{32}$. We did not observe a correlation between MPO-DNA and nucleosome levels in this study, indicating that the 
nucleosomes in these patients are probably cell-free DNA originating of other cell death mechanisms than neutrophils in NETosis. In sepsis patients, severe tissue and organ damage are the main cause of death. Cell-free DNA is therefore very likely originating from these damaged cells.

We have shown in a pilot experiment that $N$. meningitidis isolated from sepsis patients, are able to induce NETosis in neutrophils in vitro. This is in line with a previous study on NETs formation by $N$. meningitidis ${ }^{28}$. This article describes that $N$. meningitidis is also able to evade NETs different from $S$. aureus, which excretes nucleases to break down the NETs ${ }^{33} . N$. meningitidis secretes small outer membrane vesicles (SOMVs) that bind to the NETs, blocking the binding of the bacteria themselves. In our experiments, we observed that after 30-40 minutes, neutrophils underwent necrosis next to NETosis, as expected since the bacteria are known to be toxic. Altogether, our results indicate that $N$. meningitidis has developed multiple mechanisms to avoid and destroy neutrophils, which might contribute to the severe pathogenesis of these bacteria in sepsis.

This is the largest prospective cohort of children with severe meningococcal disease of which detailed clinical and extensive laboratory data are available. Although a total number of 60 patients might be relatively small compared to adult sepsis cohorts, the advantage from our study is that these children are all affected by the same bacterium, $N$. meningitidis. The detailed assays performed and measurements of several factors over time allowed us to study correlations with markers assessed in both the current and previous studies ${ }^{26,31}$. Also, we were able to follow these patients over time and collect data on many variables, including NETs and inflammatory markers.

We have shown that $N$. meningitidis is capable of inducing NETosis in neutrophils isolated from healthy adult donors in vitro. Unfortunately age matched donors were not available, as volumes obtained for diagnostic tests are limited in children. Therefore we cannot exclude that neutrophils of 
children might react differently ${ }^{34}$. However, as NETs were present in our patient serum samples, such a difference in vitro is not expected.

\section{Conclusions}

N. meningitidis are strong NETs inducers. Children admitted to PICU for meningococcal sepsis have higher NETs levels at admission and after 24 hours than controls. NETs levels were not associated with positive or negative outcome or other inflammatory markers, indicating that the beneficial and detrimental effects of NETosis in meningococcal sepsis might be balanced. We also did not find a correlation between MPO-DNA and nucleosomes, indicating that nucleosomes are not only NETs but all cell free DNA.

\section{Acknowledgements}

We would like to thank Debby Priem-Visser for her help with the ELISAs and Myrna E. Aulia for contributing to data collection. We thank Clementien Vermont, Ester de Kleijn and Marieke den Brinker for inclusion of patients and sample and data collection. Furthermore we thank N.A. Lemmens-den Toom and M. van Westreenen for culturing N. meningitidis and for their advice regarding working with this pathogen. 


\section{References}

1 Zeerleder S, Hack CE, Wuillemin WA. Disseminated intravascular coagulation in sepsis. Chest. 2005; 128: 2864-75.

2 Pace D, Pollard AJ. Meningococcal disease: clinical presentation and sequelae. Vaccine. 2012; 30 Suppl 2: B3-9.

3 Wright $V$, Hibberd $M$, Levin $M$. Genetic polymorphisms in host response to meningococcal infection: the role of susceptibility and severity genes. Vaccine. 2009; 27 Suppl 2: B90-102.

4 Emonts M, Hazelzet JA, de Groot R, Hermans PW. Host genetic determinants of Neisseria meningitidis infections. Lancet Infect Dis. 2003; 3: 565-77.

5 Loh E, Kugelberg E, Tracy A, et al. Temperature triggers immune evasion by Neisseria meningitidis. Nature. 2013; 502: 237-40.

6 Bianchi M, Hakkim A, Brinkmann V, et al. Restoration of NET formation by gene therapy in CGD controls aspergillosis. Blood. 2009; 114: 2619-22.

7 Fuchs TA, Abed U, Goosmann $C$, et al. Novel cell death program leads to neutrophil extracellular traps. J Cell Biol. 2007; 176: 231-41.

8 Brinkmann V, Reichard U, Goosmann C, et al. Neutrophil extracellular traps kill bacteria. Science (New York, NY. 2004; 303: 1532-5.

9 McDonald B, Urrutia R, Yipp BG, Jenne CN, Kubes P. Intravascular neutrophil extracellular traps capture bacteria from the bloodstream during sepsis. Cell Host Microbe. 2012; 12: 324-33.

10 Hoppenbrouwers T, Autar ASA, Sultan AR, et al. In vitro induction of NETosis: comprehensive live imaging comparison and systematic review. PLoS One. 2017.

11 Camicia G, Pozner R, de Larranaga G. Neutrophil extracellular traps in sepsis. Shock. 2014; 42: 286-94.

12 Kaplan MJ, Radic M. Neutrophil extracellular traps: double-edged swords of innate immunity. J Immunol. 2012; 189: 2689-95.

13 Czaikoski PG, Mota JM, Nascimento DC, et al. Neutrophil Extracellular Traps Induce Organ Damage during Experimental and Clinical Sepsis. PLoS One. 2016; 11: e0148142.

14 McDonald B, Davis RP, Kim SJ, et al. Platelets and neutrophil extracellular traps collaborate to promote intravascular coagulation during sepsis in mice. Blood. 2017.

15 Gould TJ, Vu TT, Stafford AR, et al. Cell-Free DNA Modulates Clot Structure and Impairs Fibrinolysis in Sepsis. Arterioscler Thromb Vasc Biol. 2015; 35: 2544-53.

16 Hermans PW, Hibberd ML, Booy R, et al. 4G/5G promoter polymorphism in the plasminogenactivator-inhibitor-1 gene and outcome of meningococcal disease. Meningococcal Research Group. Lancet. 1999; 354: 556-60.

17 Kornelisse RF, Hazelzet JA, Hop WC, et al. Meningococcal septic shock in children: clinical and laboratory features, outcome, and development of a prognostic score. Clin Infect Dis. 1997; 25: 6406.

18 de Kleijn ED, de Groot R, Hack CE, et al. Activation of protein C following infusion of protein C concentrate in children with severe meningococcal sepsis and purpura fulminans: a randomized, double-blinded, placebo-controlled, dose-finding study. Crit Care Med. 2003; 31: 1839-47.

19 Emonts $\mathrm{M}$, de Bruijne EL, Guimaraes $\mathrm{AH}$, et al. Thrombin-activatable fibrinolysis inhibitor is associated with severity and outcome of severe meningococcal infection in children. $J$ Thromb Haemost. 2008; 6: 268-76.

20 Goldstein B, Giroir B, Randolph A, International Consensus Conference on Pediatric S. International pediatric sepsis consensus conference: definitions for sepsis and organ dysfunction in pediatrics. Pediatr Crit Care Med. 2005; 6: 2-8.

21 Pollack MM, Ruttimann UE, Getson PR. Pediatric risk of mortality (PRISM) score. Crit Care Med. 1988; 16: 1110-6. 
22 Khemani RG, Bart RD, Alonzo TA, Hatzakis G, Hallam D, Newth CJ. Disseminated intravascular coagulation score is associated with mortality for children with shock. Intensive Care Med. 2009; 35: 327-33.

23 Couto-Alves A, Wright VJ, Perumal K, et al. A new scoring system derived from base excess and platelet count at presentation predicts mortality in paediatric meningococcal sepsis. Crit Care. 2013; 17: R68.

24 Borissoff JI, Joosen IA, Versteylen MO, et al. Elevated levels of circulating DNA and chromatin are independently associated with severe coronary atherosclerosis and a prothrombotic state. Arteriosclerosis, thrombosis, and vascular biology. 2013; 33: 2032-40.

25 Masuda S, Nakazawa D, Shida $\mathrm{H}$, et al. NETosis markers: Quest for specific, objective, and quantitative markers. Clin Chim Acta. 2016; 459: 89-93.

26 Bongers TN, Emonts $M$, de Maat MP, et al. Reduced ADAMTS13 in children with severe meningococcal sepsis is associated with severity and outcome. Thromb Haemost. 2010; 103: 1181-7.

27 Hoppenbrouwers T, Autar ASA, Sultan AR, et al. In vitro induction of NETosis: Comprehensive live imaging comparison and systematic review. PLoS One. 2017; 12: e0176472.

28 Lappann M, Danhof S, Guenther F, Olivares-Florez S, Mordhorst IL, Vogel U. In vitro resistance mechanisms of Neisseria meningitidis against neutrophil extracellular traps. Mol Microbiol. 2013; 89: 433-49.

29 Hashiba M, Huq A, Tomino A, et al. Neutrophil extracellular traps in patients with sepsis. J Surg Res. 2015; 194: 248-54.

30 Varju I, Longstaff C, Szabo L, et al. DNA, histones and neutrophil extracellular traps exert antifibrinolytic effects in a plasma environment. Thromb Haemost. 2015; 113: 1289-98.

31 Zeerleder S, Stephan F, Emonts $\mathrm{M}$, et al. Circulating nucleosomes and severity of illness in children suffering from meningococcal sepsis treated with protein C. Crit Care Med. 2012; 40: 3224-9.

32 Iba T, Miki T, Hashiguchi N, Tabe $\mathrm{Y}$, Nagaoka I. Is the neutrophil a 'prima donna' in the procoagulant process during sepsis? Crit Care. 2014; 18: 230.

33 Thammavongsa V, Missiakas DM, Schneewind O. Staphylococcus aureus degrades neutrophil extracellular traps to promote immune cell death. Science. 2013; 342: 863-6.

34 Simon AK, Hollander GA, McMichael A. Evolution of the immune system in humans from infancy to old age. Proc Biol Sci. 2015; 282: 20143085. 


\section{Figure legends}

Figure 1: Unstimulated neutrophils (A) vs NETs (see arrows) formed by N. meningitidis (B). Blue: Hoechst staining for DNA. Red: PI staining for extracellular DNA (NETs) and dead cells.

Figure 2: MPO-DNA levels measured at different time points (A) and in relation to survival (B). 